\title{
VLBI STUDIES OF HIGH-REDSHIFT 21CM ABSORPTION LINES
}

\author{
F. H. Briggs \\ University of Pittsburgh \\ Pittsburgh, PA 15260 USA
}

VLBI experiments can contribute to our understanding of absorbing gas observed in the spectra of high-redshift quasars by measuring the spatial extent of the absorbers. An optical survey conducted by Wolfe, Turnshek, Smith, and Cohen (1986) has turned up a class of absorbers characterized by large HI column density and a rich spectrum of metal absorption lines. In these respects the absorbers resemble spiral galaxies, but their occurence is too frequent for interception probabilities based on galaxy cross sections at the present epoch. Recent radio observations to determine the nature of such an absorber at $\mathrm{z}=2.04$ in the spectrum of PKS0458-020 include both continuum observations at 606 and $1590 \mathrm{MHz}$ to define the structure of the background quasar as well as spectral line VLBI at $467 \mathrm{MHz}$. The observations reveal structure in the continuum source on a wide range of angular scales, making the source nearly ideal for spectral line VLBI on a range of baselines. Early spectroscopic results show that the absorber must have a spatial extent of galaxian size, consistent with the hypothesis that these absorbers are galaxies. The further implication is that galaxies must have been larger and richer in gas at the epoch around $z=2$ in order to provide the likelihood of interception that is observed optically. (A complete description of the result is in preparation by Briggs, Wolfe, Liszt, Davis and Turner.)

VLBI has also proved to be a useful technique for performing spectral line studies at frequencies that fall outside the bands protected for radio astronomy: The systems studied often have redshifts that places the absorption lines in heavily used communications bands. High-redshift, radio-loud quasars often have compact structure that remains unresolved on long baselines, permitting VLBI observations that are immune to moderate levels of interference which is local to the individual stations and does not cohere in the final cross-correlation spectrum.

\section{REFERENCES}

Wolfe, A.M., Turnshek, D.A., Smith, H.E., and Cohen, R.D. 1986, Ap. J. Suppl., 61, 249. 